\title{
Agrobacterium-mediated transformation of a low glutelin mutant of 'Koshihikari' rice variety using the mutated-acetolactate synthase gene derived from rice genome as a selectable marker
}

\author{
Yuhya Wakasa $\cdot$ Kenjirou Ozawa $\cdot$ Fumio Takaiwa
}

Published online: 15 March 2008

(C) Springer-Verlag 2008

Erratum to: Plant Cell Rep (2007) 26:1567-1573

DOI 10.1007/s00299-007-0373-0

Unfortunately, the presentation of Table 1 contains errors.

The correct Table 1 is given below.

Table 1 Culture media used for callus induction, callus selection, and shoot regeneration

\begin{tabular}{|c|c|}
\hline N6D & $\begin{array}{l}\text { 4.0 } \mathrm{g} \mathrm{l}^{-1} \mathrm{CHU}^{*} 1 \mathrm{basal} \text { salt mixture (Sigma, Gillingham, UK), N6-vitamins*1, } 2 \mathrm{mg} \mathrm{l}^{-1} \text { 2,4-D, } 30 \mathrm{~g} \mathrm{l}^{-1} \text { sucrose, } 100 \mathrm{mg} \mathrm{l}^{-1} \mathrm{myo}^{-} \\
\text {inositol, } 300 \mathrm{mg} \mathrm{l}^{-1} \text { casamino acids, } 2.88 \mathrm{~g} \mathrm{l}^{-1} \text { proline, } 4 \mathrm{~g} \mathrm{l}^{-1} \text { gelrite }(\mathrm{pH} 5.8)\end{array}$ \\
\hline 2N6-AS & 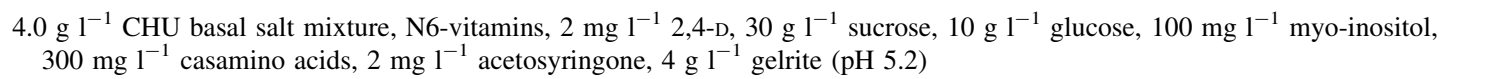 \\
\hline MS-regeneration & $\begin{array}{l}4.6 \mathrm{~g}^{-1} \mathrm{MS}^{*} 2 \text { basal salt mixture (Nihon Pharmaceutical, Tokyo, Japan), B5-vitamins*3, } 0.2 \mathrm{mg} \mathrm{l}^{-1} \mathrm{NAA}, 2 \mathrm{mg} \mathrm{l}^{-1} \text { kinetin, } \\
30 \mathrm{~g} \mathrm{l}^{-1} \text { sucrose, } 30 \mathrm{~g} \mathrm{l}^{-1} \text { sorbitol, } 2 \mathrm{~g} \mathrm{l}^{-1} \text { casamino acids, } 4 \mathrm{~g} \mathrm{l}^{-1} \text { gelrite (pH 5.8) }\end{array}$ \\
\hline Modified-DKN & $\begin{array}{l}\text { DKN-Macro*4, DKN-Micro*4, R2-Iron*5, B5-vitamins, } 2 \mathrm{mg} \mathrm{l}^{-1} \text { 2,4-D, } 30 \mathrm{~g} \mathrm{l}^{-1} \text { sucrose, } 100 \mathrm{mg} \mathrm{l}^{-1} \text { mio-inositol, } 300 \mathrm{mg}^{-1} \\
\text { casamino acids, } 1.15 \mathrm{~g}^{-1} \text { proline, } 2 \mathrm{mg} \mathrm{l}^{-1} \text { glycine, } 670 \mathrm{mg} \mathrm{l}^{-1} \text { L-aspartic acid, } 730 \mathrm{mg}^{-1} \text { L-glutamine, } 4 \mathrm{~g} \mathrm{l}^{-1} \text { gelrite }(\mathrm{pH} \text { 5.8) }\end{array}$ \\
\hline DKN-AS & $\begin{array}{l}\text { DKN-Macro, DKN-Micro, R2-Iron, B5-vitamins, } 2 \mathrm{mg}^{-1} \text { 2,4-D, } 30 \mathrm{~g} \mathrm{l}^{-1} \text { sucrose, } 10 \mathrm{~g} \mathrm{l}^{-1} \text { glucose, } 100 \mathrm{mg} \mathrm{l}^{-1} \text { myo-inositol, } \\
670 \mathrm{mg} \mathrm{l}^{-1} \text { L-aspartic acid, } 730 \mathrm{mg} \mathrm{l}^{-1} \text { L-glutamine, } 10 \mathrm{mg} \mathrm{l}^{-1} \text { acetosyringone, } 4 \mathrm{~g} \mathrm{l}^{-1} \text { gelrite ( } \mathrm{pH} \mathrm{5.2)}\end{array}$ \\
\hline $\begin{array}{r}\text { Modified-DKN } \\
\text { regeneration }\end{array}$ & $\begin{array}{l}\text { DKN-Macro, DKN-Micro, R2-Iron, B5-vitamins, } 0.2 \mathrm{mg} \mathrm{l}^{-1} \mathrm{NAA}, 2 \mathrm{mg} \mathrm{l}^{-1} \text { kinetin, } 30 \mathrm{~g}^{-1} \text { sucrose, } 30 \mathrm{~g} \mathrm{l}^{-1} \text { sorbitol, } 100 \mathrm{mg}^{-1} \\
\text { myo-inositol, } 2 \mathrm{~g} \mathrm{l}^{-1} \text { casamino acids, } 2 \mathrm{mg}^{-1} \text { glycine, } 670 \mathrm{mg} \mathrm{l}^{-1} \mathrm{~L} \text {-aspartic acid, } 730 \mathrm{mg} \mathrm{l}^{-1} \text { L-glutamine, } 4 \mathrm{~g} \mathrm{l}^{-1} \text { gelrite } \\
\text { (pH 5.8) }\end{array}$ \\
\hline MS-hormon free & 4.6 $\mathrm{g} \mathrm{l}^{-1} \mathrm{MS}$ basal salt mixture, B5-vitamins, $30 \mathrm{~g} \mathrm{l}^{-1}$ sucrose, $2.5 \mathrm{~g} \mathrm{l}^{-1}$ gelrite ( $\mathrm{pH} 5.8$ ) \\
\hline
\end{tabular}

*1 Chu et al. (1975), *2 Murashige and Skoog (1962), *3 Gamborg et al. (1968), *4 Daigen et al. (2000), *5 Ohira et al. (1973)

The online version of the original article can be found under doi:10.1007/s00299-007-0373-0.

Y. Wakasa $\cdot$ F. Takaiwa $(\bowtie)$

Transgenic Crop Research and Development Center,

National Institute of Agrobiological Sciences, Kannondai 3-1-3,

Tsukuba, Ibaraki 305-8602, Japan

e-mail: takaiwa@nias.affrc.go.jp

Y. Wakasa

e-mail: ywakasa@nias.affrc.go.jp

K. Ozawa

Department of Low-Temperature Sciences,

National Agricultural Research Center for Hokkaido Region,

Hitsujigaoka, Toyohira-ku, Sapporo, Hokkaido 062-8555, Japan

e-mail: ozawa@affrc.go.jp 